\title{
Evaluation of Serum Amyloid A3 in Young Adult Males with Early Onset of Androgenic Alopecia
}

A.M.El-Refaie, E.M.Akl, W.A.Abdelhalim and M.M.Mahmoud

${ }^{1}$ Dermatology, Venerology and Andrology Dept., Faculty of Medicine, Benha Univ., Benha, Egypt

${ }^{2}$ Clinical and Chemical Pathology Dept., Faculty of Medicine, Benha Univ., Benha, Egypt

E-Mail:Mai.Mahmoud@gmail.com

\begin{abstract}
Androgenetic alopecia (AGA) which is also known as androgenic alopecia or male pattern baldness, is the most common type of progressive hair loss. Male androgenetic alopecia (MAA) is the most common form of hair loss in men, affecting 30$50 \%$ of men by age 50. Male androgenetic alopecia (MAA) occurs in a highly reproducible pattern, preferentially affecting the temples, vertex and mid frontal scalp.To estimate the Serum Amyloid A3 (SAA3) in young male patients with AGA and studying the relationship between the level of SAA3 and atherosclerosis. The investigation was done after endorsement by the Ethics advisory group on Research at Faculty of Medicine, Benha University. A composed educated assent was acquired from every member. This examination was a cross sectional case similar investigation. All patients chose from Outpatient Clinic of Dermatology, Venerology and Andrology Department of Benha University Hospitals. This examination was begun at January 2019 to June 2019. We found that the patients with grade III and IV androgenetic alopecia with family ancestry of heart illnesses and raised lipid profile levels had critical height of serum amyloid A3 than different gatherings. Male patients underneath age of 30 years with AGA exceptionally grade III, IV had raised SAA3 and have more dangers to create blood vessel atherosclerotic illness later on than the people without AGA at a similar age gathering.
\end{abstract}

Keywords: Androgenetic alopecia, Male androgenetic alopecia, Cardiovascular disease, Ischemic heart disease.

\section{Introduction}

Androgenetic alopecia (AGA) is one of the most widely recognized introducing issue at dermatology centers (40-half of guys at fourth or fifth decades) [14].

Male androgenetic alopecia (MAA) is the most widely recognized type of balding in men, influencing 30-half of men by age 50. Male androgenetic alopecia (MAA) happens in a profoundly reproducible example, specially influencing the sanctuaries, vertex and mid frontal scalp [4].

Androgenetic alopecia (AGA) is brought about by a dynamic decrease in the width, length and pigmentation of the hair. Hair diminishing outcomes from the impacts of the testosterone metabolite dihydrotestosterone (DHT) on androgen-delicate hair follicles [15].

Various examinations have explored a likely relationship among AGA and cardiovascular sickness (CVD) by investigating restricted and scattered boundaries [5].

Male AGA is acquired as a complex polygenic quality. It has been exhibited that extreme beginning stage of AGA in youthful subject ( $<30$ years) have an expanded danger of Ischemic coronary illness (IHD). An examination demonstrated that men with higher evaluations of AGA (vertex), have a higher danger of creating IHD, particularly among men having elevated cholesterol level and hypertension [17].

High androgen level adds to the improvement of atherosclerosis, apoplexy prompting hypertension and hypercholesterolemia [2].

Atherosclerosis is an interminable aggravation related with expanded articulation of the intense stage isoforms of serum amyloid A (SAA) and in people is a plasma biomarker for future cardiovascular occasions. In any case, regardless of whether SAA is just a biomarker or takes part in the improvement of cardiovascular illness isn't very much portrayed [6].
Fat tissue secretes proteins like serum amyloid A (SAA), which assumes significant jobs in neighborhood and foundational aggravation. SAA3 mRNA articulation in intra-stomach fat were fundamentally higher in fat human [3].

Serum amyloid A (SAA) predicts cardiovascular occasions. Over articulation of SAA expands atherosclerosis advancement; be that as it may, inadequacy of two of the murine intense stage isoforms, SAA1.1 and SAA2.1, has no impact on atherosclerosis. SAA3 is a pseudogene in people however it is a communicated intense stage isoform in mice [19].

\section{Aim of the work}

This present study aimed to estimate the Serum Amyloid A3 (SAA3) in young male patients with AGA and studying the relationship between the level of SAA3 and atherosclerosis.

\section{Patients and methods Moral contemplations}

The investigation was done after endorsement by the Ethics board of trustees on Research at Faculty of Medicine, Benha University. A composed educated assent was acquired from every member.

\section{Sort of the investigation}

This examination was a cross sectional case relative examination.

\section{Study area}

All patients chose from Outpatient Clinic of Dermatology, Venerology and Andrology Department of Benha University Hospitals.

\section{Study length}

This investigation was begun at January 2019 to June 2019 
Study populace: This examination included 80 guys separated into two gatherings: (Group An) included 50 youthful grown-up guys younger than 30 years of age with androgenetic alopecia. (Gathering B) included 30 clearly solid age coordinated males(under the age of 30 years of age) as a benchmark group.

Consideration Criteria: Under the age of 30 years of age. All had clinical image of various degrees of androgenetic alopecia.

Prohibition measures: Above 30 years of age. With fundamental ailments (hepatic, renal and immune system maladies). Patients who had chemotherapy. Endocrinal illnesses (Thyroid, pituitary or adrenal issue) were rejected from the examination by history. Patients taking any hormonal strengthening (Androgen, against androgen or insulin sharpening drugs). Inflammatory or irresistible maladies in scalp were prohibited by assessment. Alopecia areata and scarring alopecias, which can emulate AGA.

\section{Control Group}

Age coordinated gathering will be: Under age of 30 years of age. No foundational sicknesses like(hepatic ailments, renal infection, auto aimmune illness, endocrinal malady). No different cutaneous illnesses.

\subsection{Methods}

Every patient was exposed to the accompanying:

Full history taking: A deliberately structured sheet was performed for all patients and controls in this investigation, including: Personal history: Name, sex, age, occupation, and extraordinary propensities like smoking. Present history: Onset, length and course of male pattern baldness. Patients were requested introductory indications of AGA like tingling. Previous history of medication consumption: An assortment of medications that can cause balding were gotten some information about like, chemotherapeutic specialists (practically all of which can create male pattern baldness) and for hormones with genius androgenic or antithyroid activity. Especially in men, the admission of anabolic steroids or supplemental androgens is investigated. Way of life methods, for example, exceptional hairdos (footing) and ecological variables like smoking and bright radiation introduction were considered throughout the entire existence of the patients. Family ancestry for AGA, DM and hypertension.

Physical assessment: Clinical assessment included the scalp skin and hair, facial and body hair.

Scalp assessment: Signs of inflammation (erythema, scaling and hyperkeratosis), seborrhoea and indications of scarring (decay, loss of hair follicle ostia) were thought of.

Scalp hair assessment: Hamilton-Norwood scale is utilized to assess male example dispersion. In most of men, AGA includes the fronto-transient and the vertex regions, and male pattern baldness advances to end-stage blading in 50-60\% of men by age of 70 years Fig (7) [19]. This procedure may begin whenever following adolescence or sooner. The most far reaching use characterization technique for male AGA is the one that portrayed by Norwood in 1975, with refining Hamilton's grouping [20].

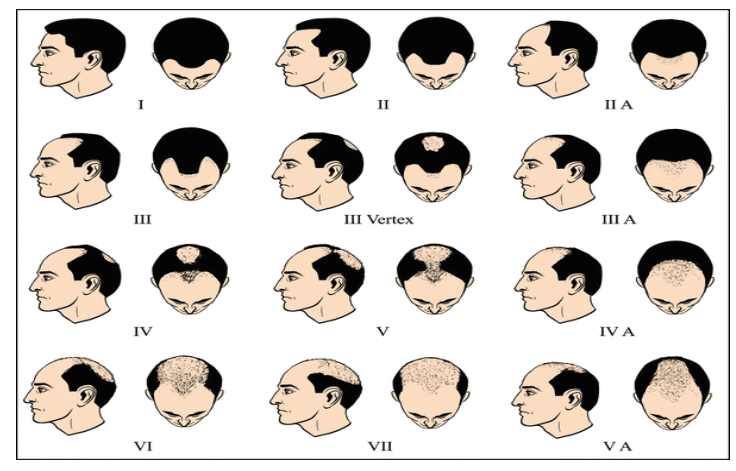

Fig (1) Hamilton-Norwood classification of male balding [20].

Facial and body hair

Facial and body hair were examined for abnormal facial and body hair density and/or distribution.

\section{Clinical anthropometry}

Weight. Height. BMI. The BMI was calculated by dividing body weight by the square of the height $\left(\mathrm{kg} / \mathrm{m}^{2}\right)$. Midsection and hip estimation. Midriff circuit was estimated at the midpoint of the tightest part between the base of the rib confine and the highest point of the iliac peak while the patient was standing erect, with the midregion loose, arms along the edges and feet together, with weight similarly isolated over the two legs. Hip boundary was recorded as the greatest outline over the bum.
BP estimation: For BP estimation, a sphygmomanometer was utilized. BP was resolved in a calm domain after the patient had stayed situated for 10 min. All members had advanced photography in three distinct projections and two separate dermatologists played out a blinded evaluation of the seriousness of alopecia.

Research center examinations: Amyloid A3 was estimated utilizing Elisa packs (201-12-1257) from Shanghai Sunred Biological Technology Company China, in view of the rule of twofold counter acting agent sandwich procedure to recognize Human serum amyloid A3(SAA3).Add SAA3 to monoclonal neutralizer Enzyme well which is precoated with Human serum amyloid A3monoclonal immunizer, hatching; at that point, include 
SAA3 antibodies named with biotin, and joined with Streptavidin-HRP to shape safe complex; at that point complete brooded and washing again to evacuate the uncombined protein. At that point include chromogen arrangement A, B, the shade of the fluid changed into the blue, and at the effect of corrosive, the shading at long last got yellow.

\subsection{Statistical analysis}

The clinical data were recorded on a report form. These data were tabulated and analysed using the computer program SPSS (Statistical package for social science) version 20 to obtain:

\section{Descriptive data}

Table (1) Comparison between patients and control group regarding age.

\begin{tabular}{|c|c|c|c|c|c|c|}
\hline & \multicolumn{2}{|c|}{ Case group (50) } & \multicolumn{2}{|c|}{ Control group (30) } & \multirow{2}{*}{$\begin{array}{c}\text { Statistical } \\
\text { test }\end{array}$} & \multirow[t]{2}{*}{ P value } \\
\hline & Mean & \pm SD & Mean & \pm SD & & \\
\hline Age (years) & 28.0 & 2.26 & 27.3 & 1.82 & St $\mathrm{t}=1.44$ & 0.154 \\
\hline BMI $\left(\mathrm{Kg}_{\mathrm{g}} \mathrm{m}^{2}\right)$ & 26.26 & 2.81 & 24.2 & 1.85 & $\mathrm{St} \mathrm{t}=3.58$ & $0.001 * *$ \\
\hline
\end{tabular}

Table (2) Clinical data of all patients. qualitative data.
Descriptive statistics were calculated for the data in the form of: Mean and standard deviation $( \pm S D)$. for quantitative data. Frequency and distribution for

\section{Analytical statistics}

In the statistical comparison between the different groups, the significance of difference was tested using one of the following tests Student's $t$-test:- Used to compare mean of two groups of quantitative data. ANOVA (F) test:-Used to compare mean of more than two groups of quantitative data. Inter-group comparison of categorical data was performed by using chi square test $\left(X^{2}\right.$-value).

\section{Results}

\begin{tabular}{|c|c|c|}
\hline \multirow{3}{*}{ Age (years) mean \pm SD } & \multicolumn{2}{|c|}{ The studied group (80) } \\
\hline & \multicolumn{2}{|c|}{ Value } \\
\hline & 27.61 & 2.16 \\
\hline 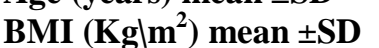 & 25.49 & 2.67 \\
\hline \multicolumn{3}{|l|}{ Family history (FH) n(\%) } \\
\hline Positive & 43 & 53.8 \\
\hline Negative & 37 & 46.2 \\
\hline \multicolumn{3}{|l|}{ Consanguinity $\mathbf{n}(\%)$} \\
\hline Positive & 19 & 23.8 \\
\hline Negative & 61 & 76.2 \\
\hline \multicolumn{3}{|c|}{ History of cardiac disease $n(\%)$} \\
\hline Positive & 32 & 40.0 \\
\hline Negative & 48 & 60.0 \\
\hline
\end{tabular}

Table (3) Family history of AGA, consanguinity, history of cardiac disease, blood pressure and pulse between patients and controls.

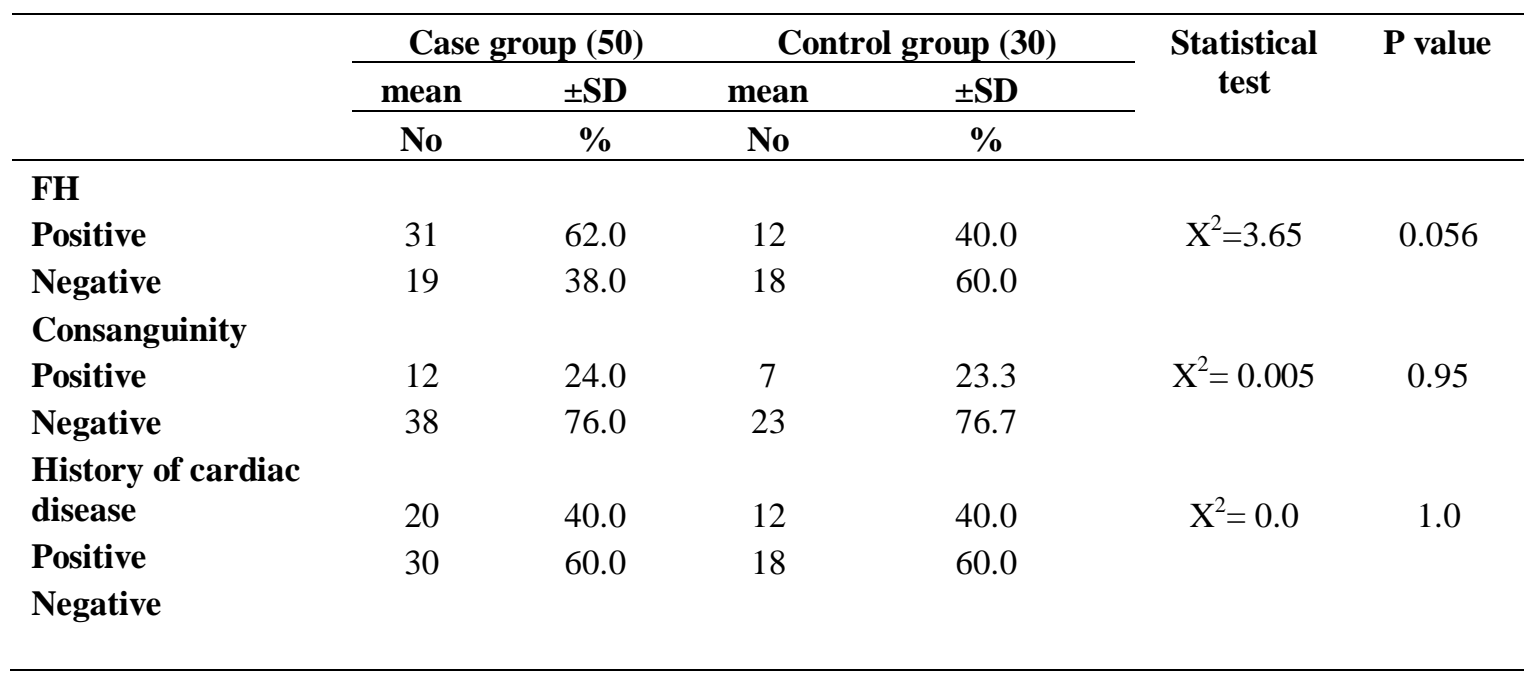


Table (3) Continue

\begin{tabular}{lcccccc}
\hline & mean & \pm SD & mean & \pm SD & St t test & \\
SBP & 132.9 & 9.64 & 130.0 & 7.99 & 1.39 & 0.17 \\
DBP & 80.0 & 6.62 & 78.83 & 5.52 & 0.81 & 0.42 \\
Pulse & 80.28 & 8.44 & 77.13 & 8.45 & 1.61 & 0.111 \\
\hline
\end{tabular}

\section{Discussion}

Androgenetic alopecia (AGA) which is otherwise called androgenic alopecia or male example sparseness, is the most widely recognized sort of dynamic male pattern baldness. AGA is a polygenetic condition with differing seriousness, period of beginning, and scalp area of male pattern baldness. In men, balding normally includes the fleeting and vertex area while saving the occipital locale [12].

Male androgenetic alopecia (MAA) is the most wellknown type of male pattern baldness in men, influencing 30 -half of men by age 50. Male androgenetic alopecia (MAA) happens in an exceptionally reproducible example, specially influencing the sanctuaries, vertex and mid frontal scalp [4].

In spite of the fact that the commonness is high in old patients, AGA may likewise begin at adolescence. Patients who have conspicuous diminishing of hair are seen as more seasoned, which influences confidence and prompts psychosocial horribleness. Rather than the high commonness of AGA, affirmed restorative alternatives are constrained [20].

The utilization of the clinical term androgenetic alopecia reflects current information about the significant job of androgens and hereditary factors in AGA etiology. It has for quite some time been realized that the nearness of testosterone in hair follicles is a pre-requisite for AGA, while hereditary elements alter the greatness of the hair follicle reaction to flowing androgens [16].

Polymorphism of the androgen receptor quality (AR) was first recognized in relationship with AGA. The confusing job of androgens in hair follicle (HF) science remains inadequately saw; to be sure, androgens trigger hair improvement at adolescence and contrarily favor AGA in later life [13].

Androgenetic alopecia is androgen needy and most presumably a polygenic issue with an inherited inclination. Polygenic heredity is thought to be the essential driver, in spite of the fact that the male hormone testosterone assumes a significant job. In the hair follicle cells, testosterone changes over into the naturally progressively dynamic metabolite, dihydrotestosterone because of compound 5- $\alpha$ reductase [9].

It has been accepted that androgenetic alopecia is the aftereffect of the unusual affectability of hair follicles of the scalp to flowing androgens and expanding in the quantity of androgen receptors. The chemical 5-alpha reductase has two isoforms, types 1 and 2, which catalyze the change of testosterone to 5-alpha-dihydrotestosterone. It is accepted that both isoforms assume a job in the digestion and activity of androgen, and their appearance differs relying upon the site of the body [8]. S. Liu et al. [11] found a higher articulation of 5-alpha reductase type 1 in hair follicles, proposing that they assume a key job in androgen-directed hair development.

Androgenetic alopecia in male creating before multi year old enough with at any rate Grade III of HamiltonNorwood grouping is named as beginning stage or untimely AGA. Men with untimely AGA are seen as vulnerable to cardiovascular maladies, metabolic disorder (MS), diabetes mellitus, hypertension, and furthermore untimely hair loss can have a positive negative effect on the mental self view and confidence in these patients [18].

High androgen level adds to the advancement of atherosclerosis, apoplexy prompting hypertension and hypercholesterolemia [2].

A relationship between metabolic condition and beginning stage androgenetic alopecia may add to the inclination of patients with androgenetic alopecia to create cardiovascular illness [7].

Serum amyloid A (SAA), an intense stage lipoprotein, has been read for a considerable length of time as a vigorous biomarker for a wide exhibit of provocative and immune system issue. Numerous isoforms of this protein have been distinguished, including: SAA1 and H.S. Banger et al. [2], which are profoundly homologous and transcendently delivered by the liver; SAA3, an intensely communicated isoform created in non-primate warm blooded animals [1].

J.C. Thompson [19] found in their investigation that SAA3 is professional atherogenic in mice and they suggested that the ceaseless height of SAA in people with conditions, for example, stoutness, metabolic disorder, and diabetes might be a hidden reason for the expanded cardiovascular malady saw in these populaces.

Metabolic disorder (MetS) is a bunch of between related hazard factors that expansion the danger of coronary vein illness (CAD) and incorporates focal stoutness, hypertension, hyperglycemia, and dyslipidemia [21].

Atherosclerosis is a constant irritation related with expanded articulation of the intense stage isoforms of serum amyloid A (SAA) and in people is a plasma biomarker for future cardiovascular occasions [6].

In this way, this current investigation meant to gauge the Serum Amyloid A3 (SAA3) in youthful male patients with AGA and considering the connection between the degree of SAA3 and atherosclerosis.

This examination was case controlled investigation and all patients were chosen from outpatient center of Dermatology and Andrology division of Benha University Hospitals from March 2019 to July 2019.

The investigation was affirmed by the nearby morals board of trustees on research including human subjects, Faculty of Medicine, Benha University. 
This investigation was led on 50 youthful male patients experiencing AGA (Group A) their mean time of was $28.0 \pm 2.26$ years old. What's more 30 obviously solid people of coordinated age and sex were picked as control gathering (Group B) their mean age was $27.3 \pm 1.82$ years old. Every patient was exposed to full history taking, total general and dermatologic assessment estimating BMI, BP, FBG, Lipid profile, reviewing AGA and estimation the degree of serum amyloid A3 (SAA3) utilizing Elisa procedure.

In our examination, we found that there were factually critical contrast among patients and controls in BMI, which concurred with the investigation done by [18].

Additionally, by examination the family ancestry of AGA and helplessness of transmission to the cutting edge in cases and controls bunches we found that the cases had solid connection between their AGA and their family ancestry showed up as $62 \%$ of the cases had positive family ancestry of AGA while $38 \%$ of it had negative family ancestry of AGA while in control bunch we found that $40 \%$ of controls had positive family ancestry and $60 \%$ of it had negative family ancestry.

In spite of the fact that, there were increment in family ancestry of cardiovascular sickness in cases than controls however this expanding had no critical distinction.

There were no factually huge contrasts among patients and controls in both systolic and diastolic Blood Pressure.

The outcomes demonstrated that there were critical contrasts among cases and controls with respect to fasting blood glucose (FBG) and LDL. The mean FBG in cases was $127.2 \mathrm{mg} / \mathrm{dl}$ which was higher than control gathering. There were no critical contrasts among cases and controls in regards to HDL, TGS and all out cholesterol.

Metabolic condition noted in patients with high FBG and lipid profile more than control which concurred with the consequences of [7] and this may clarify that this gathering is more obligated to have atherosclerosis than others.

Atherosclerosis is the basic reason for coronary episode and stroke. Early perceptions that cholesterol is a key part of blood vessel plaques offered ascend to the cholesterol speculation for the pathogenesis of atherosclerosis. Populace contemplates have shown that raised degrees of LDL cholesterol and apolipoprotein B, the fundamental basic protein of LDL, are legitimately connected with hazard for atherosclerotic cardiovascular occasions [10]. So in this current examination the patients which had higher noteworthy LDL progressively obligated to have atherosclerosis sickness.

The current examination results indicated that serum AA3 articulation was essentially higher in AGA patients than controls. Additionally the outcomes indicated that the degree of serum AA3 was high in patients with positive family ancestry of AGA, positive association and patients with III and IV evaluations of AGA. Likewise serum AA3 was somewhat high in patients with metabolic disorder. This outcomes imply that patients with AGA who have serum amyloid A3 increasingly obligated to have metabolic condition and atherosclerotic sicknesses.
Serum AA3 was demonstrated no noteworthy distinction among the diverse benchmark groups with various circumstances in regards to the family ancestry, relationship, lipid profile and history of cardiovascular maladies.

Along these lines, from this investigation we uncovered that the youthful male patients before the age of thirty years of age with AGA (principally grade III and IV) have more hazard to create future blood vessel atherosclerotic infection and the hazard will increment when related with metabolic condition and solid family ancestry of constant illnesses, this was finished by evaluating the serum amyloid A3 (SAA3) in plasma which consider professional atherogenic marker and discovered measurably huge contrast and height of the serum amyloid A3 (SAA3) in youthful male patients with AGA more than non-AGA youthful people.

Along these lines, we can utilize the serum amyloid A3 (SAA3) as early pointer marker to the future blood vessel atherosclerotic infection in youthful AGA since it has cut off worth about(1.81) with high affectability (90\%) and explicitness $(80 \%)$ and exactness was $86 \%$ which involves the serum amyloid A3 (SAA3) in early discovery in blood vessel atherosclerotic sickness in youthful male patients with AGA.

\section{Conclusion}

Male patients below age of 30 years with AGA specially grade III, IV had elevated SAA3 and have more risks to develop arterial atherosclerotic disease in the future than the persons without AGA at the same age group.

\section{References}

[1] J.L. Ather , M.E. Poynter,Serum amyloid A3 is required for normal weight and immunometabolic function in mice. PLoS One, Vol. 13(2),PP.e0192352,2018.

[2] H.S. Banger, S.K. Malhotra, S. Singh, Is early onset androgenic alopecia a marker of metabolic syndrome and carotid artery atherosclerosis in young Indian male patients?. International journal of trichology, Vol. 7(4), PP.141,2015.

[3] T. Chiba, C.Y. Han, T. Vaisar, Serum amyloid A3 does not contribute to circulating SAA levels. Journal of lipid research, Vol.50(7), PP.1353-62,2009.

[4] W. Cranwell , R. Sinclair , Male androgenetic alopecia. InEndotext [Internet]. MDText. com, Inc, Vol. 53(2),PP. 52-25,2016

[5] R. Ertas, O. Orscelik, D. Kartal, Androgenetic alopecia as an indicator of metabolic syndrome and cardiovascular risk. Blood pressure, vol,25(3), PP.141-8, 2016.

[6] G.S. Getz, P.A. Krishack , C.A. Reardon, Serum amyloid $\mathrm{A}$ and atherosclerosis. Current opinion in lipidology, Vol.27(5), PP. 531-535, 2009.

[7] H. Gopinath , G.M. Upadya , Metabolic syndrome in androgenic alopecia. Indian J Dermatol Venereol Leprol, Vol.82, PP.404-8,2016. 
[8] L. Jacobo, C.D. Villarreal-Villarreal , R. Ortiz-López, Genetic and molecular aspects of androgenetic alopecia. Indian J Dermatol Venereol Leprol, Vol.84, PP. 263-8, 2018.

[9] Z. Kolar, R. Kucerova ,M. Beinova, Androgenetic alopecia and polymorphism of the androgen receptor gene (SNP rs6152) in patients with benign prostate hyperplasia or prostate cancer JEADV , Vol.25(2), PP.91-96,2014.

[10] M.F. Linton, P.G. Yancey , S.S. Davies, The Role of Lipids and Lipoproteins in Atherosclerosis, Vol.30(4), PP. 425-430, 2019.

[11] S. Liu, H. Yamauchi, Different patterns of 5alphareductase expression, cellular distribution, and testosterone metabolism in human follicular dermal papilla cells. Biochem Biophys Res Commun, Vol.368(4), PP. 858-64, 2008.

[12] F. Lolli, F. Pallotti, A. Rossi, Androgenetic alopecia: a review. Endocrine, Vol.57(1), PP. 9-17,2017.

[13] L. Michel, P. Benech, P. ReygagneStudy of gene expression alteration in male androgenetic alopecia: evidence of predominant molecular signalling pathways. British Journal of Dermatology (BJD), vol 27(3),PP. 1322-1336, 2017.

[14] T. Parviz, M. Sanei Taheri, O. Eslamnia , Androgenic Alopecia and Subclinical Atherosclerosis: Is Any Relationship? Journal of Clinical \& Experimental Dermatology Research, Vol.8(05), PP. 1-3, 2017.
[15] B.M. Piraccini, A. Alessandrini , Androgenetic alopecia. G Ital Dermatol Venereol. , Vol.149(1), PP. 15-24, 2017.

[16] A. Rossi, A. Anzalone , M.C. Fortuna, Multi therapies in androgenetic alopecia. Review and clinical experiences. Dermatol ther, Vol. 29(5), PP. 424-432, 2016.

[17] K.H. Sharma , A. Jindal, Association between androgenetic alopecia and coronary artery disease in young male patients. International journal of trichology, Vol. 6(1), PP.5, 2014.

[18] M.R. Swaroop, B.M. Kumar,P. Kumari, The Association of Metabolic Syndrome and Insulin Resistance in Early-Onset Androgenetic Alopecia in Males: A Case-Control Study. Indian J Dermatol, Vol.64(1),PP. 23-27,2019.

[19] J.C. Thompson, P.G. Wilson , P. Shridas, Serum amyloid A3 is pro-atherogenic. Atherosclerosis, Vol.268, PP. 32-35,2018.

[20] S. Varothai ,W.F. Bergfeld ,Androgenetic alopecia: an evidence-based treatment update. Am J Clin Dermatol,Vol.15(3), PP. 217-30,2014.

[21] R.V. Vora, G. Anjaneyan , R.R. Singhal, Clinical Profile of Androgenic Alopecia and Its Association with Cardiovascular Risk Factors. Indian J Dermatol, Vol. 64(1), PP. 19-22,2014. 